\title{
Compact, ultra-low vibration, closed-cycle helium recycler for uninterrupted operation of MEG with SQUID magnetometers
}

\author{
Chao Wang ${ }^{1 *}$, Limin Sun ${ }^{2}$, Ben Lichtenwalter ${ }^{1}$, Brent Zerkle ${ }^{1}$, and Yoshio Okada ${ }^{2}$ \\ ${ }^{1}$ Cryomech, Inc., 113 Falso Drive, Syracuse, NY 13211, USA \\ ${ }^{2}$ Boston Children's Hospital and Harvard Medical School, 300 Longwood Ave., Boston, MA \\ 02115, USA
}

\section{Abstract}

A closed-cycle helium recycler was developed for continuous uninterrupted operation for magnetometer-based whole-head magnetoencephalography (MEG) systems. The recycler consists of a two stage $4 \mathrm{~K}$ pulse-tube cryocooler and is mounted on the roof of a magnetically shielded room (MSR). A flexible liquid helium ( $\mathrm{LHe}$ ) return line on the recycler is inserted into the fill port of the MEG system in the MSR through a slotted opening in the ceiling. The helium vapor is captured through a line that returns the gas to the top of the recycler assembly. A highpurity helium gas cylinder connected to the recycler assembly supplies the gas, which, after it is liquefied, increases the level of LHe in the MEG system during the start-up phase. No storage tank for evaporated helium gas nor a helium gas purifier is used. The recycler is capable of liquefying helium with a rate of $\sim 17 \mathrm{~L} / \mathrm{d}$ after precooling the MEG system. It has provided a fully maintenance-free operation under computer control for 7 months without refill of helium. Although the recycler is used for single-orientation operation at this initial testing site, it is designed to operate at +/-20 degree orientations, allowing the MEG system to be tilted for supine and reclining positions. Vibration of the recycler is dampened to an ultra-low level by using several vibration isolation methods, which enables uninterrupted operation during MEG measurements. Recyclers similar to this system may be quite useful even for MEG systems with $100 \%$ magnetometers.

*Corresponding author: Chao Wang, Cryomech, Inc., 113 Falso Drive, Syracuse, NY 13211, USA, email: cwang@cryomech.com 


\section{Keywords (5 keywords max)}

Closed-cycle helium recycler; magnetoencephalography (MEG); pulse-tube cryocooler, superconducting quantum interference device (SQUID), Gifford-McMahon cryocooler

\section{Abbreviations}

MEG - magnetoencephalography; SQUID - superconducting quantum interference device; MSR

- magnetically shielded room; BCH - Boston Children's Hospital; PPMS - physical property

management system; MPMS - magnetic property measurement system; GM - Gifford-

McMahon cryocooler; JT- Joule-Thomson cryocooler; MRI - magnetic resonance imaging; LHe - liquid helium

\section{Introduction}

Magnetoencephalography (MEG) is a functional neuroimaging technique for mapping brain activity by recording magnetic fields produced by electrical currents occurring naturally in the brain. All of the commercial MEG systems (i.e. Elekta-Neuromag VectorView and Triux; CTF MEG; Tristan MagView) presently use superconducting detection coils inductively coupled to superconducting quantum interference devices (SQUIDs) to measure MEG signals. SQUIDs are extremely sensitive detectors of magnetic flux. They utilize the Josephson junction to detect MEG signals from the human brain varying between $10^{-11}$ and $10^{-14}$ Tesla.

All of these MEG systems operate at the temperature of liquid helium (LHe) (4 Kelvin), requiring LHe as the coolant. In many MEG facilities, the evaporating helium gas is released from the cryostat of the MEG system into the atmosphere, requiring regular manual transfer of the cryogen into the MEG system. This mode of operation is quite costly since the supply of LHe is scarce, expensive and sometimes unreliable, and the regular helium transfer consumes a significant amount of labor of a trained technician or technicians. Since helium is a precious natural resource that cannot be manufactured, some facilities use a method to capture the evaporating vapor and recondensing the gas. Although this second mode of operation saves helium, it limits the use of MEG to only large universities or research facilities which consume a 
significant amount of helium to justify the operation of a sophisticated and expensive reliquefaction facility. The large expense of helium maintenance has limited a wide-spread use of this technology for measuring human brain functions.

On-site helium recovery has recently become recognized as necessary in the field of MEG technology as helium becomes more expensive and an off-site recovery system may be difficult to establish in many cases. In response, some manufacturers have developed an open-cycle recovery system that collects the gas and reliquefies and stores LHe in a tank onsite. The stored LHe is then transferred back into the MEG cryostat in a conventional manner. Although this method recovers most (>80-90\%) of helium, it still requires manual transfer of LHe by a skilled technician or technicians.

In order to eliminate the manual transfer altogether, closed-cycle recyclers are beginning to be developed for MEG facilities. In one approach, the recycler is placed above MEG sensors within a single cryostat in order to reduce the heat loss from the recycler to the MEG cryostat. Sata et al $[1,2]$ have shown that the recycler noise produced by a GM/JT recycler can be reduced to $15 \mathrm{pT}$, sensed by axial gradiometers with a detection coil diameter of $20 \mathrm{~mm}$ and a baseline of $50 \mathrm{~mm}$. Using a template matching noise rejection algorithm, the noise of the recycler $(2 \mathrm{~Hz}$ with harmonics) could be reduced to below the system noise level (white noise level 12-18 fT/ $\sqrt{\mathrm{Hz}}$ ) to measure evoked averaged MEG signals from human volunteers. Yu et al [3] have used a similar design with the cold-head of the cryocooler inside the MEG cryostat. A superconducting plate was successfully used $25 \mathrm{~mm}$ above the MEG magnetometer array to reduce the noise from the cryocooler to as little as $1.7 \mathrm{pT} / \sqrt{\mathrm{Hz}}$ at the center of the superconductor plate. The superconductor plate produces an image of each magnetometer on the opposite side of the plate to provide an effective noise cancellation using this image [4]. More recently, a closed-cycle recycler has been developed by Elekta-Neuromag in Helsinki, Finland. In their system, a GT cold head is installed in the cryostat of the whole-head MEG system so that it can be tilted for measurements with the subject on a reclined seat or on a bed. The recycler, however, must be turned off during MEG measurements.

In the second approach, the recycler is placed outside the MEG cryostat to reduce its noise seen by the MEG sensors. One challenge for this approach is reduction of LHe loss in the transfer tube from the recycler to the MEG cryostat. Takeda et al $[5,6]$ have developed a closedcycle recycler using two 1.5W@4.2K GM cryocoolers with a multi-concentric transfer tube. This 
recycler uses dual helium streams, one to collect evaporated helium vapor and to return it as liquefied helium to the MEG cryostat, and the second to use higher temperature helium gas $(\sim 40 \mathrm{~K})$ to cool the radiation shield. Their recycler can liquefy at $\sim 35.5 \mathrm{~L} / \mathrm{d}$. Although MEG measurements can be carried out while the recycler is operating, the noise from the GM pump causes a large amount of interference that requires an elaborate vibration damping method to reduce the noise. More recently, Adachi et al [7] have developed a closed-cycle recycler using a pulse tube that produces an order of magnitude less vibration noise than the GM recyclers. Using a principal component analysis (PCA)-based noise cancellation algorithm, the recycler noise was reduced to below $50 \mathrm{fT} / \sqrt{ } \mathrm{Hz}$.

We have developed an efficient, closed-cycle helium recycler for MEG that is compact and simple in design without requiring a storage gas tank and a gas purifier as in other systems, with a very low vibration noise for uninterrupted operation of the recycler during MEG measurements. Unlike all previous attempts, we have successfully developed a system that can be used for MEG systems consisting entirely of magnetometers. This recycler uses a single, two-stage pulse tube cryocooler that has been developed at Cryomech [8,9]. The recycler has been used for some open cryostats, such PPMS and MPMS, wet dilution refrigerators and superconducting magnets etc. Below, we describe the design of this system and its basic performance characteristics using a small test reservoir. Then, we describe the performance of this system installed at a whole-head MEG facility of Boston Children's Hospital $(\mathrm{BCH})$ recently established for basic and clinical human brain development research. We show that the recycler noise is sufficiently small so that the residual noise can be eliminated with well-established, simple noise cancellation algorithms and spontaneous brain activity measured by a magnetometer array can be monitored online in real time while the recycler is operating continuously uninterrupted. The performance of our recycler is overall superior to all previous closed-cycle systems.

\section{System design}

The recycler developed in this project is an extension of our previous recyclers based on a two-stage $4 \mathrm{~K}$ pulse tube cryocooler - Cryomech model PT415RM $(1.5 \mathrm{~W}$ at $4.2 \mathrm{~K})[8,9]$. The development of a recycler specifically for MEG systems called for a modification of the basic system to significantly reduce vibration noise and to eventually allow for use with two different orientations of the MEG cryostat with the subject in the supine and reclined seated positions. The 
vibration was significantly reduced through two major steps. First, the motor/rotary valve assembly is detached from the pulse tube cold head. The vibration transmission from the motor to the cold head is minimized using a flexible tube. Second, the transmission of vibration from the cryocooler to the MEG system was reduced by using a flexible transfer tube connecting the recycler to the fill port of the MEG cryostat.

Figure 1 shows the design of the recycler. The high-pressure helium gas from the compressor is delivered to the rotary valve-motor assembly (4) via a pair of long flexible tubes (3). The motor assembly is detached from the cold head (5) to reduce vibration of the recycler assembly (7). It is mounted on a sliding carriage (2). The sliding carriage allows free movement of the remote motor to reduce the force which could exert on the recycler assembly. A similar design has been used in a dry dilution refrigerator [10]. The rotary valve feeds compressed gas to the cold head (5) via a flexible line (4). A bellows (6) is inserted between the cold head and the recycler assembly to reduce vibration transmission. A support structure (12) mounted on the ceiling (d) holds the cold head firmly. 
The cold head and recycler assembly are shown in some detail on the right. The recycler contains a 2 -stage pulse tube $(15,16)$ encased in a vacuum insulated sleeve (17). A thermal shield (18) is used to reduce infrared radiation penetrating the vacuum container. The reliquefied LHe drips down through the flexible transfer tube (8) (444 mm long and $47 \mathrm{~mm}$ OD) and an insertion tube (9) (660 mm long and $12.7 \mathrm{~mm} \mathrm{OD}$ ) into the inner belly (a) of the MEG cryostat (b). The flexible part of the liquid return tube accommodates the MEG rotation up to 20 degrees and also reduces the vibrations transferred from the recycler assembly to the MEG (b). The evaporating helium vapor returns to the recycler via two paths: (1) through the transfer tube which allows for LHe to drip down and helium gas to rise up to the recycler chamber through a single tube without vapor trap; and (2) through a vapor line (11), made of stainless steel flexible
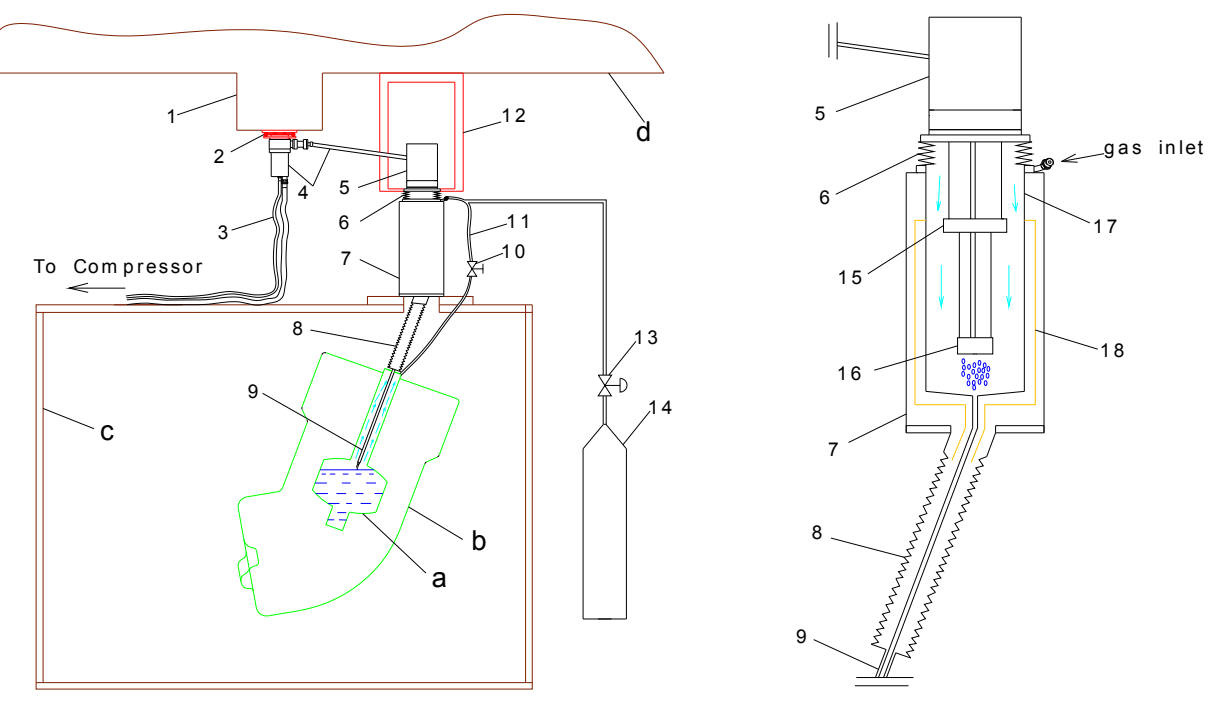

Fig. 1. System design for the closed-loop, noise-free, continuous operation recycler for MEG installed in the clinical MEG facility of Boston Children's Hospital. (Left) Schematics of the system design. (Right) Schematics of the recycler design. (a) liquid helium reservoir of MEG; (b) MEG; (c) magnetic shielded room (MSR); (d) ceiling. (1) Mounting base for the remote motor/rotary valve assembly; (2) sliding carriage; (3) flexible lines between compressor and rotary valve; (4) remote motor/rotary valve assembly; (5) Cold head of pulse tube cryocooler; (6) vibration isolation bellows; (7) recycler assembly; (8) flexible part of liquid return line; (9) insertion part of liquid return line; (10) needle valve; (11) vapor line; (12) support structure of the cold head; (13) pressure regulator; (14) gas cylinder; (15) $1^{\text {st }}$ stage of the cold head; (16) 2nd stage of the cold head; (17) vacuum insulated sleeve; (18) radiation shield of the recycler assembly. 
line, with a needle valve (10) to the gas inlet at the top of the recycler. In the recycler assembly (7), the pulse tube cold head resides in the vacuum insulated sleeve (17). Via path 1, LHe drains back to the belly of the MEG reservoir (a) through the liquid return line $(8,9)$. The helium vapor/gas via path 2 is pre-cooled by the first stage, the tubes of regenerators and pulse tubes, and finally condensed on the $2^{\text {nd }}$ stage/condenser. A ultra high purity helium gas cylinder (14) is attached to the same gas inlet with a flow valve (13) to allow for building up the LHe level during the start-up phase. The recycler assembly is firmly mounted on the roof of the magnetically shielded room (MSR) for damping most vibrations from the recycler assembly.

\section{Experimental results and analysis}

\subsection{Recycler performance test}

The recycler performance was first evaluated at Cryomech prior to installing the recycler at the MEG facility at $\mathrm{BCH}$. The recycler was connected to a $\mathrm{LHe}$ reservoir of $1.4 \mathrm{~L}$ with its radiation shield cooled by vapor [8]. The recycler was tilted by $20^{\circ}$ for this evaluation since it was designed to be used with MEG systems that can be tilted by $+/-20^{\circ}$.

The performance of the PT415RM pulse tube cryocooler was measured first before integrating it into the recycler assembly. Figure 2 shows the cooling performance of the

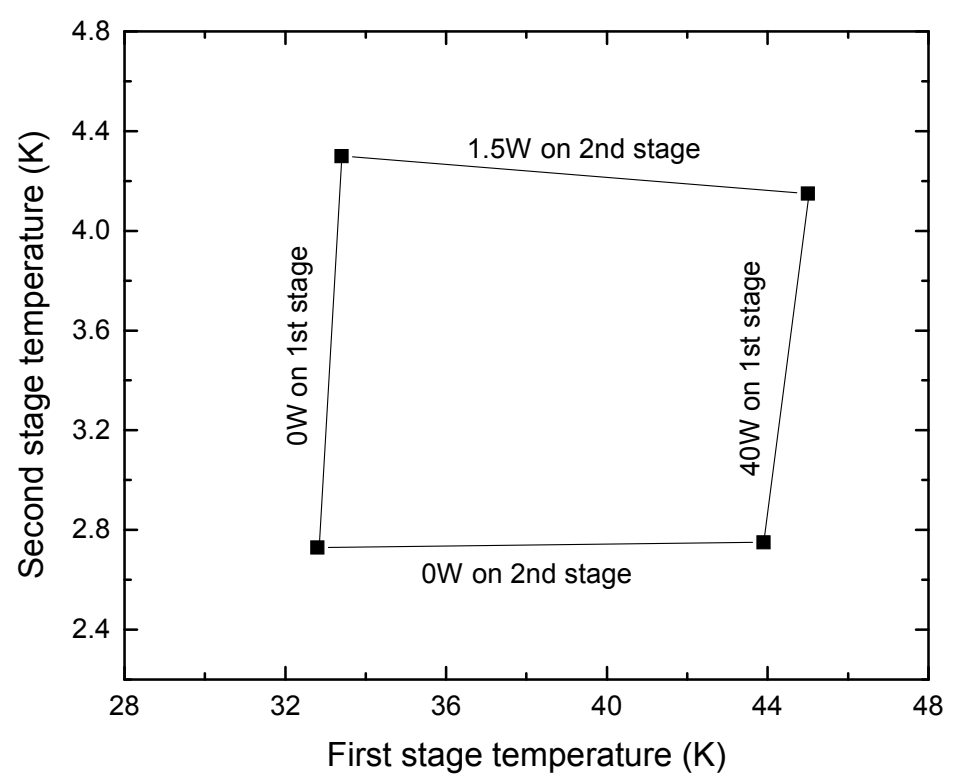

Fig. 2. Measured cooling load map of the PT415RM 
PT415RM at different temperatures. It can provide $40 \mathrm{~W}$ at $45 \mathrm{~K}$ on the $1^{\text {st }}$ stage and $1.5 \mathrm{~W}$ at $4.18 \mathrm{~K}$ on the $2^{\text {nd }}$ stage simultaneously while consuming an electrical power of $9.8 \mathrm{~kW}$.

The recycler was installed into the testing cryostat for closed-cycle operation. As in Fig. 1, the gas inlet on the top of the recycler was connected to a gas supply cylinder to provide ultra high purity helium gas to the recycler. The testing procedures were as follows:

Step 1: The recycler and the testing cryostat are cooled down from room temperature. Once the liquid reservoir in the testing cryostat reaches the temperature of $\sim 4.2 \mathrm{~K}$, it starts to collect liquid helium.

Step 2: A mass flow meter measures the flow rate of helium from the gas cylinder during the liquefaction, which indicates the liquefaction rate from room temperature gas.

Step 3: When the liquid reaches a level of $\sim 10 \mathrm{~cm}$, the helium gas supply from the gas cylinder is shut off. The heater on the liquid reservoir is turned on to simulate the heat load to the cryostat. The heater power indicates the liquid boil-off rate of the cryostat, thus, the capacity for liquefaction and recondensing.

Figure 3 shows the cool down curve of the recycler. The $2^{\text {nd }}$ stage/condenser on the cold head

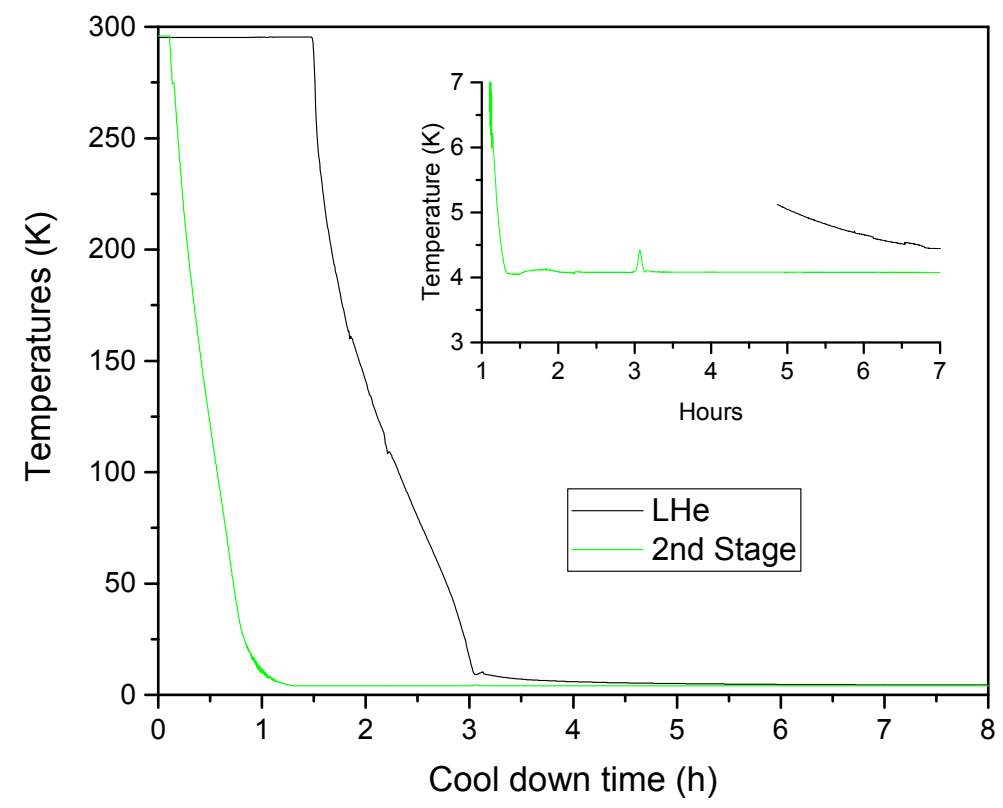

Fig. 3. Recycler cools down in the testing cryostat

quickly reaches liquefaction temperature of $\sim 4.2 \mathrm{~K}$ in 1.3 hours. The temperature of the LHe reservoir drops down to $10 \mathrm{~K}$ in about 3 hours and reaches $4.2 \mathrm{~K}$ in about 7 hours, at which time LHe starts accumulating in the reservoir. 
The gas flow rate is measured during the liquefaction process (Fig. 4). The flow rate is in the range from 7.88 to 8.47 SLPM during the measurement, corresponding to the liquefaction rate of 16.2 to $17.4 \mathrm{~L} /$ day. The variation of the liquefaction rate could be caused by the increasing temperature of the radiation shield in the testing cryostat. During the liquefaction, there is less vapor cooling through the neck of the testing cryostat, which results in a higher temperature on the radiation shield.

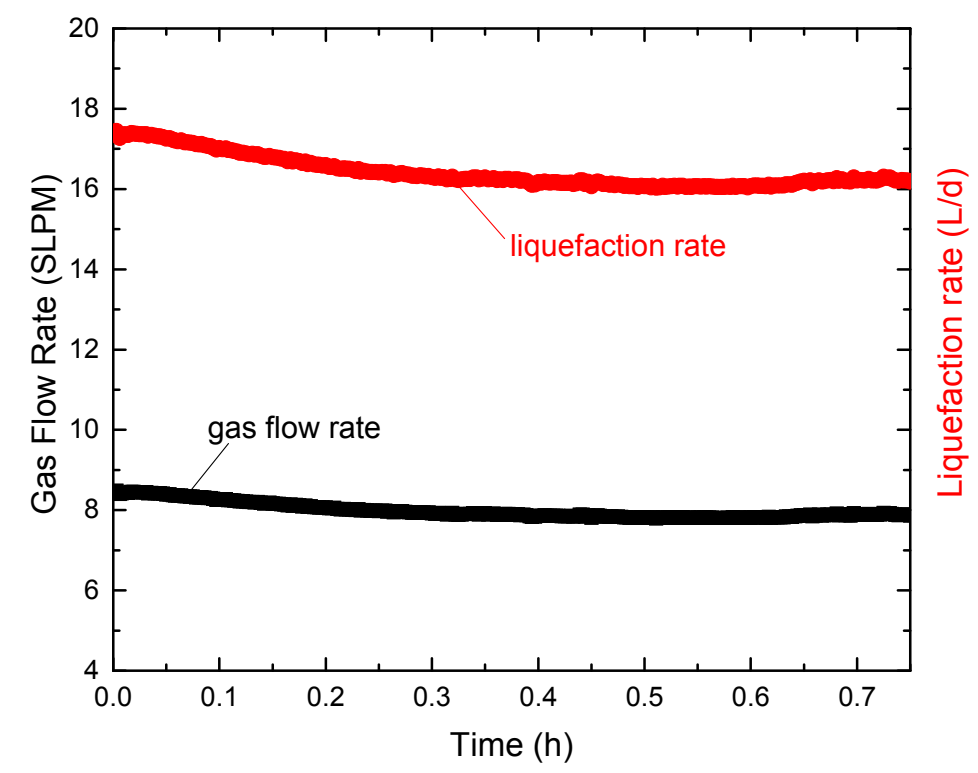

.4. Liquefaction rate of the recycler.

Fig

The helium supply is turned off when the reservoir has around $1 \mathrm{~L}$ of LHe. The heater in the liquid reservoir is turned on to simulate heat load to the liquid helium reservoir. Figure 5 shows

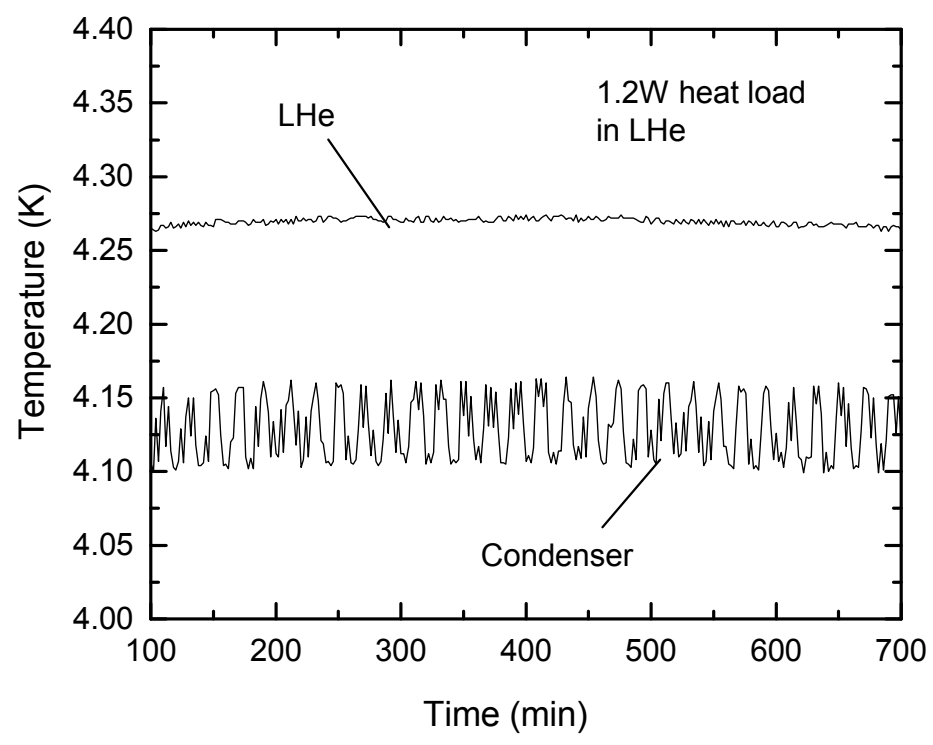

Fig. 5. Recondensing/re-liquefaction testing. 
the temperatures in the LHe and on the condenser while a heat load of $1.2 \mathrm{~W}$ is applied to the LHe. A heat load of $1.2 \mathrm{~W}$ boils off $40 \mathrm{~L}$ of LHe in a day. Due to partially recondensing the vapor of $4.2 \mathrm{~K}[12,13]$ through the liquid return line, the recycler has much higher capacity to recover the helium vapor from a cryostat. But with this function, the vapor flowing through the neck of cryostat is reduced. The reduced vapor flow rate through the neck lowers the ability of the MEG cryostat to cool the radiation shields and other components in the vacuum section. In some MEG cryostats such as the whole-head MEG system installed at $\mathrm{BCH}$ that have the MEG sensors in the vacuum section of the cryostat, this vapor cooling of the neck is very important since it is used for cooling the thermal radiation shields, which help to reduce the temperature of the MEG sensors in the vacuum section. The valve (10) on the vapor line (11) can be used to balance the vapor flow through the vapor line and liquid return line for the best system performance for such MEG systems.

\subsection{Performance of recycler in the MEG facility at Boston Children's Hospital}
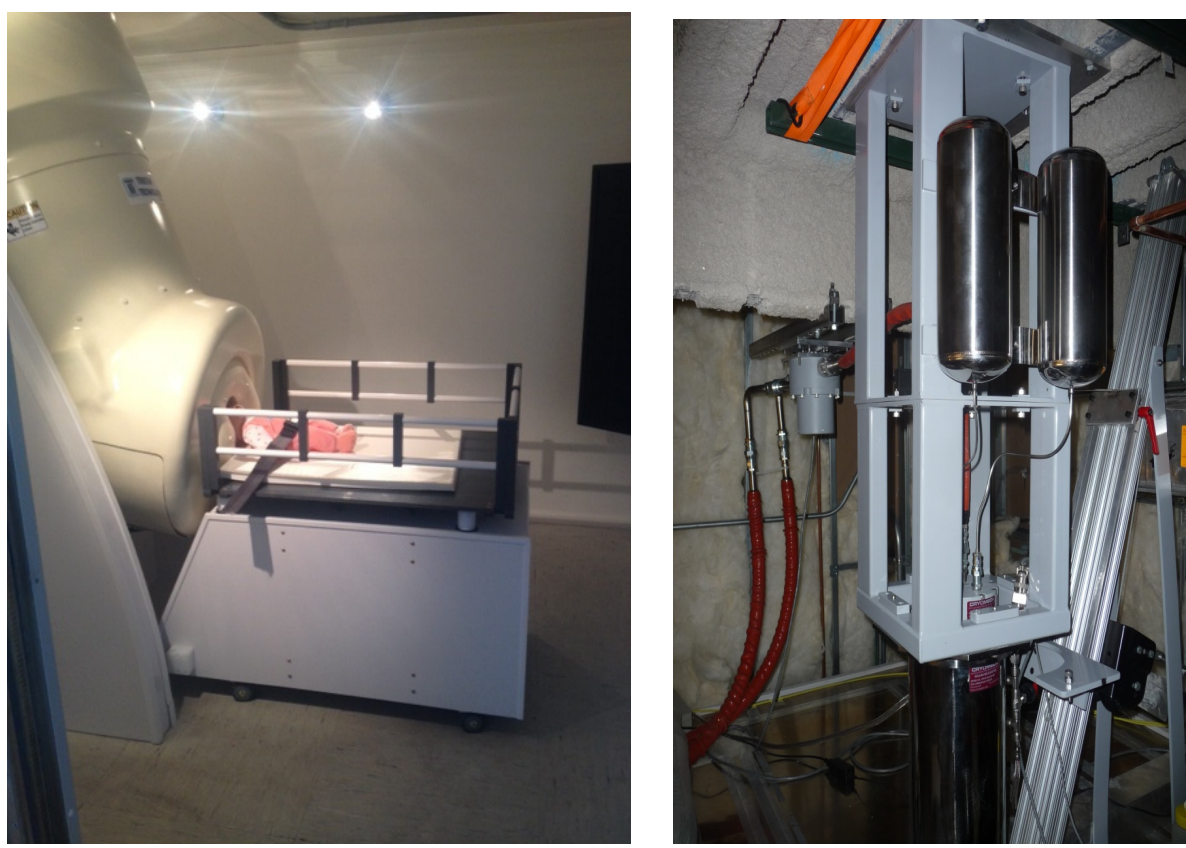

Fig. 6. Closed-cycle helium recycler installed in a pediatric MEG system. (a) The pediatric, 384-channel, whole-head MEG system installed in the clinical MEG facility at Boston Children's Hospital. (b) Recycler installed above the ceiling of the MSR. The recycler is connected to the MEG system via a flexible LHe transfer tube inserted into the neck of the MEG cryostat. 
Upon completing the initial evaluation of the recycler at Cryomech, it was installed in the MEG facility at Boston Children's Hospital. The facility has a whole-head pediatric MEG system, called "babyMEG”, constructed by Tristan Technologies (Okada et al, in preparation). The babyMEG has 270 magnetometers in the inner layer of the array 5-6 mm from the inner surface of the helmet, 35 sets of 3-axis magnetometers in the outer layer $4 \mathrm{~cm}$ away from the inner layer and 9 reference magnetometers. The array is placed in the vacuum space just above the helmet in order to achieve a minimal gap $(8-9 \mathrm{~mm})$ between the magnetometers and the scalp for an MEG system. To achieve a uniform gap all around the head, the sensor array is held by a position adjustment mechanism within the vacuum space with six rods protruding from the top plate of the cryostat for remote control of the sensor position and orientation. A bank of 384 SQUIDs are in the vacuum space around the bottom of the belly of the LHe reservoir. The SQUIDs are attached to the data processing equipment via a set of cryocables in the vacuum space. There is a dual layer thermal shield in the vacuum space.

The recycler must be capable of maintaining all of these materials at a temperature that allow the magnetometers to operate as superconducting magnetic field sensors and maintain the level of LHe in the reservoir at a fixed steady level (30 L capacity). The vibration noise from the recycler must be sufficiently low to provide facile noise cancellation in real time during MEG measurements. Furthermore, the recycler should operate maintenance free for many months. The system described here is capable of achieving these goals.

At BCH, an off-the-shelf pulse tube recycler based on Cryomech PT410 was installed first in order to evaluate its performance and to identify how the recycler needs to be modified for the final system. This initial recycler was configured as in Fig. 1 without any storage tank for excess helium gas evaporating from the MEG system and without a helium gas filter in the input line to the stage I of the cold head. The transfer tube was a standard tube available at Cryomech - it was not flexible except at the base for orienting the tube at $20^{\circ}$ from vertical. The recycler with the cold head was placed on the roof of the MSR with the transfer tube inserted through a slot in the ceiling of the MSR into the neck and belly portion of the cryostat. The compressor, which provides pressurized helium gas to the pulse tube cold head, is placed on the floor just outside the MSR.

This initial system showed that the vibration noise was small enough to operate the recycler even during MEG measurements, by using the noise cancellation algorithm described below. 
However, it could not produce enough LHe to maintain the helium level ( $\sim 13-14 \mathrm{~L} / \mathrm{d}$ evaporation rate for this BabyMEG). Small leaks were identified at the joints in several ports on the top plate of the MEG system, which had to be eliminated. We also discovered that it was necessary to maximize the flow of the evaporating helium gas through the neck area of the inner storage LHe storage tank (a in Fig. 1) in order to cool the massive amount of materials in the vacuum section of the cryostat. The thermal shield between the sensor array and the helmet had to be cooled to $<67 \mathrm{~K}$ for the magnetometers to function as superconducting sensors. Thus, the end of the transfer tube $(8,9)$ inside the belly was lengthened using a G-10 extension tube of $210 \mathrm{~mm}$ long. It minimized the vapor flowing through the liquid return line when the end of the liquid return was submerged in the LHe. The needle valve (10) was opened nearly fully to maximize the flow of the cold vapor through the neck area of the reservoir. The need for maximizing the vapor pressure in the neck area also led to a change in the control point used for maintaining the gas pressure level in the recycler system. A heater was inserted in the top portion of the belly of the MEG system to control the gas pressure inside the recycler. The second and final recycler was designed and constructed based on our experience using the first system. We describe the performance of this final system next.

\subsubsection{Performance of the recycler after an initial stage of precooling}

During the initial evaluation, the recycler performance was always tested after precooling the MEG system using the conventional method of LHe transfer through an input port on the top plate of the MEG cryostat and building up a level of LHe in the reservoir.

However, in one instance, we evaluated the power of this recycler to liquefy helium from zero helium level in the cryostat. At the start, the MEG system was at room temperature, according to the thermometers installed at strategic positions in the vacuum space. LHe was transferred to the reservoir via the conventional method using a $100 \mathrm{~L}$ helium tank. This precooling constricted all the components in the cryostat including the reservoir and the micropositioning device attached to the sensor array. The constriction helped to create a gap between the helmet, the thermal shield around the inner surface of the helmet and the sensor array next to the thermal shield. This removed the physical contact between these layers to allow cooling of the thermal shield and sensor array without injecting excessive heat into the vacuum section. The precooling also reduced the vacuum pressure by at least three orders of magnitude to $<10^{-5}$ mbar due to the 
presence of molecular sieves in the vacuum section. These two factors were crucial for facilitating the cooling of the system with the recycler.

Figure 7 shows that this recycler was capable of liquefying helium from zero LHe at the start. The figure indicates the temperature at the bottom of the belly of the LHe reservoir in the MEG system and accumulation of LHe in the reservoir as a function of time after the recycler was turned on. It took nearly 90 hours for the belly temperature to reach $<10 \mathrm{~K}$ due to the large amount of material in the vacuum section of this MEG system. At $\sim 140$ hours after the start, LHe started to accumulate. Due to low flow rate from the helium gas cylinder, the accumulation of LHe stopped during period c. After adjusting the gas flow, the level started increasing again during period $\mathrm{d}$ and it reached the final maintenance level during period e. 


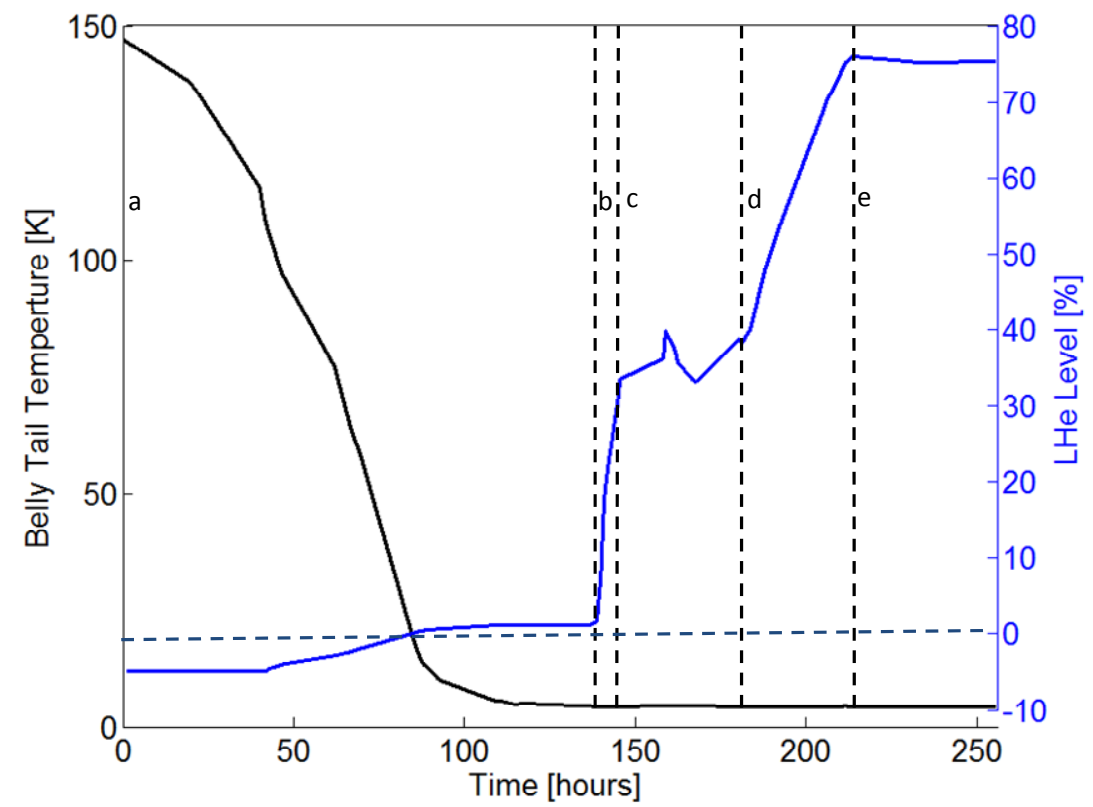

Fig. 7. Recycler can accumulate LHe in the whole-head, 384-channel MEG system after initial precooling of the bottom of the belly of the $30 \mathrm{~L}$ LHe reservoir of the MEG system to $\sim 150 \mathrm{~K}$. Belly temperature at left and LHe level in the reservoir at right. a - initial cooling with the recycler. $\mathrm{b}$ - accumulation of LHe in the reservoir. $\mathrm{c}$ - insufficient helium gas in the system. $\mathrm{d}$ - increase in LHe level with extra helium gas fed from the gas cylinder. e - steady-state, maintenance-level helium. Note: LHe level was below zero and slightly above zero before LHe accumulation at $\sim 140 \mathrm{hrs}$ due to helium level meter false reading in the absence of LHe.

\subsubsection{Performance of the MEG system during continuous, uninterrupted recycler operation}

Once the recycler operation and all the temperatures of the components of the MEG system became stable, the magnetic noise produced by the recycler was evaluated. Figure 8 shows the amplitude spectral density of the output of one of the magnetometers of the inner layer of the MEG system with the cryocooler ON and OFF. When the cryocooler was off, the noise level is $\sim 7 \mathrm{fT} / \sqrt{ } \mathrm{Hz}$ (median noise) at 200-400 Hz (a low-pass filter is applied with a cut-off frequency of $512 \mathrm{~Hz}$ ). The noise is higher at lower frequencies because this is the output of a magnetometer in a 2-layer MSR (Vacuumschmeltz) with a performance of 1-layer MSRs. When the cryocooler is turned on, Figure 8 shows the noise spike at the fundamental frequency of $1.4 \mathrm{~Hz}$ and its harmonics. 


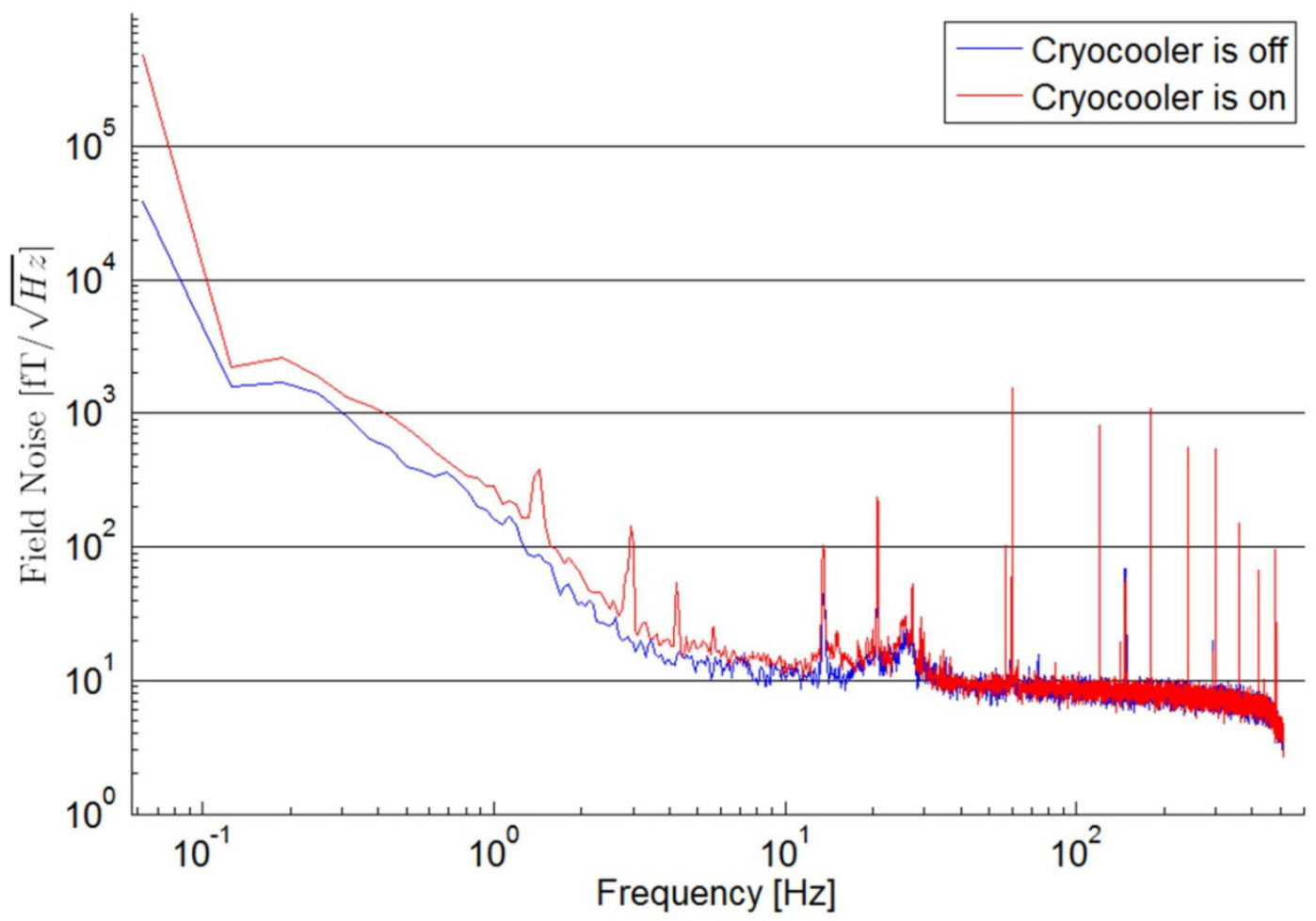

Fig. 8. Raw output of a magnetometer channel in the inner layer of the sensor array with and without the recycler on.

It was possible to remove all of the noise from the recycler using two simple methods of noise cancellation that took advantage of the 2-layer, whole-head sensor array design of this MEG system. The sensor array with 270 channels in the inner layer is effective in removing the external magnetic noise, including the noise from the recycler, with the technique called signal space projection (SSP) [11]. This SSP can be used to remove the noise in real time so that the MEG system can be used for studying spontaneous and evoked brain activity without the contamination from the recycler.

The black trace in Fig. 9 is the raw data of the same channel in Fig. 8 with the recycler ON. The fundamental is at $1.4 \mathrm{~Hz}$ and its harmonics are shown in the inset. The second inset shows the noise around the line frequency of $60 \mathrm{~Hz}(56-62 \mathrm{~Hz})$. The green trace shows the noise after applying the SSP. The SSP removed some of the recycler noise as well as the line frequency noise and the broad environmental noise below $100 \mathrm{~Hz}$. 
The dual-layer sensor array design enables the use of the second approach - formation of

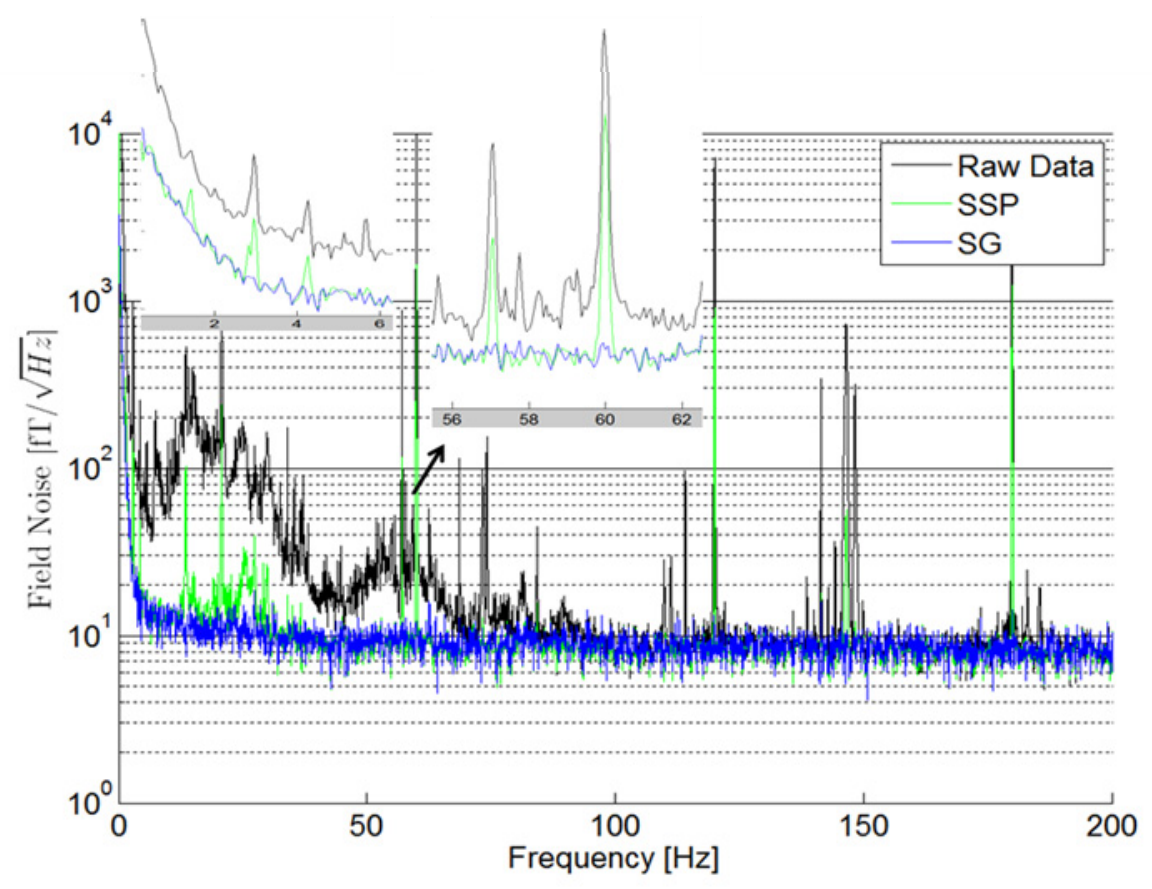

Fig. 9. Elimination of the recycler noise with the SSP and synthetic gradiometer (SG).

synthetic gradiometers (SG), by combining the output of each magnetometer in the inner layer with the outputs of all the channels in the outer layer using a Wiener filter. For each magnetometer in the inner layer, this procedure removes the component of the magnetic field that can be explained by a linearly weighted sum of the signals sensed by all the magnetometers in the outer layer. The blue trace in Fig. 9 shows that using this SG the reduction of recycler noise is further improved.

\section{Discussion}

The results demonstrate that a simple, maintenance-free, closed-cycle helium recycler can replace the conventional method of transferring LHe periodically to maintain the cryogen in an MEG system. The conventional method is costly since the cryogen is quite expensive and it requires one or more trained technicians for regularly performing the transfer. Presently, the cost of LHe is approximately $\$ 10 / \mathrm{L}$ in USA. Whole-head MEG systems consume about 8-13 L/day depending on the system, costing about $\$ 80-\$ 130 /$ day or $\$ 30,000-\$ 50,000 /$ year. The labor cost can be significant if the transfer is performed often. Most MEG sites still use this conventional method. The recycler described here is simple and maintenance free. The system does not have 
an elaborate set of helium storage tanks, a helium gas purifier, a flow pump and a mass flow controller, as used in other configurations (Elekta-Neuromag system, the system by Adachi et al [11]). The costs of liquid helium and labor are eliminated for operating the MEG system.

The recycler is capable of regenerating LHe after precooling the MEG system. It was able to start accumulating LHe in $7 \mathrm{hrs}$ in a $1.4 \mathrm{~L}$ reservoir during the initial evaluation. It was able to accumulate LHe from the starting temperature of $145 \mathrm{~K}$ at the bottom of the belly of the babyMEG. It required approximately 5 days to start accumulating LHe in the cryostat because of a large amount of material in the vacuum section of this 384-channel babyMEG. Nevertheless, it was able to accumulate LHe to the maintenance level. The precooling with conventional LHe transfer was very important since the sensors were in the vacuum section with the thermal helmet shield 1-2 mm from the inner surface of the helmet. Precooling constricted the inner container of LHe (a in Fig. 1). The sensor array and the helmet thermal shield were attached to the bottom of the belly at room temperature. This constriction moved the sensor array and the helmet shield 2-3 mm way from the helmet to eliminate physical contact between these structures. The precooling was also necessary to increase the vacuum pressure (by $>3$ orders of magnitude) beyond the level that could be achieved with a diffusion-mechanical pump system - this extra vacuum was helpful in liquefying and maintaining helium.

This recycler can be operated continuously during measurements with the recycler on, without any interference from electromagnetic and vibrational noise from the recycler. The magnetic noise level sensed by the magnetometers at the fundamental frequency of the pulse tube noise was approximately $400 \mathrm{fT} / \sqrt{ } \mathrm{Hz}$ with the recycler turned on. Separating the recycler from the MEG system and installing relatively simple methods of vibration reduction greatly reduce the noise from the recycler sensed by the MEG sensors, making the noise cancellation much easier to achieve. The noise level is $>1 \mathrm{pT}$ sensed by a gradiometer array in a recycling system with the cold head in the MEG cryostat, even after using many noise cancellation methods to reduce the noise, making it quite difficult to measure MEG signals when the recycler is on [6]. The MEG measurements cannot be carried out with the recycler on when the cold head is inside the cryostat of a whole-head MEG system (Triux, Elekta-Neuromag). The recycler noise can be reduced to below the noise level of an MEG system when the recycler is outside of the MSR as in our approach. Takeda et al $[9,10]$ have reduced the recycler noise from their GM pumps seen by a gradiometer array to below the system noise level, but they had to use an elaborate transfer 
tube and a vibration anchoring method. Adachi et al [11] have used a pulse tube, taking advantage of the smaller vibration compared to GM pumps, and have shown that their recycler can be used to reduce the noise level to $<50 \mathrm{fT} / \sqrt{ } \mathrm{Hz}$ at $\sim 3 \mathrm{~Hz}$ after eliminating the noise with a software method. However, in all these cases the vibration noise was evaluated for MEG systems with a gradiometer array. In our case, we were able to show that the recycler can be used continuously for a magnetometer-based MEG system. The noise level in our case was reduced to below the system noise level at the fundamental frequency of the recycler noise $(1.4 \mathrm{~Hz})$ and above.

This new capability greatly expands the ease of using MEG for studying human brain activity. The maintenance-free operation with zero helium cost is truly remarkable for some of us who have lived with the conventional helium transfer from the very early days of MEG research. Unlike previous recycling systems, our system easily provides a recycler noise-free operation for routine online monitoring of brain activity with the recycler operating continuously all day, every day. With the use of a recycler like the one reported here, it should be possible for a wider community of scientists to use MEG for research in basic and clinical neurosciences.

\section{Conclusion}

We have successfully designed, constructed and operated a simple, compact, maintenancefree, continuous uninterrupted operation, closed-cycle helium recycling system for whole-head MEG systems with a sensor array entirely of magnetometers, reducing the cost of helium to zero and eliminating the tedious labor of periodic manual transfer of liquid helium.

\section{Acknowledgment}

Supported by NSF grants 0958669 and 0959294, a contribution from Boston Children's Hospital (Departments of Neurology and Medicine), and a donation from Boston Investment Conference.

\section{Reference}

[1] Sata K, Fujimoto S, Fukui N, Haraguchi E, Kido T, Nishiguchi K, Kang YM. A 61-channel SQUID system for MEG meausurement cooled by a GM/JT cryocooler. IEEE Trans. Appl. Superconduct., 1997, 7: 2526-2529. 
[2] Yoshida T, Sata K, Yoshii K, Kang Y M and Suzuki A. A cryocooled 61-channel MEG system Appl Supercond. 1998, 5:385-

[3] Yu KK, Lee YH, Shim JH, Hwang SM, Kim JM, Kwon HCK, Kim KW. Closed-cycle cryocooled SQUID system with superconductive shield for biomagnetism. Supercond. Sci. Technol., 2014, 27: 105007 (9 pages).

[4] van Hulsteyn D B, Petschek A G, Flynn E R and Overton W C Jr. 1995 Superconductor imaging surface magnetometry Rev. Sci. Instrum. 1995, 66:3777-.

[5] Takeda T, Okamoto M, Atsuda K, Kobayashi A, Owaki T, Katagiri K. An efficient helium circulation system with small GM cryocoolers. Cryogenics, 2008, 48: 6-11.

[6]Takeda T, Okamoto M, Atsuda K, Katagiri K. Performance of a Helium Circulation System for a MEG, Cryogenics, 2009, 49: 144-150.

[7] Adachi Y, Oyama D, Kawai J, Uehara G, Fujihira J, Fujihira H. Low noise closed-cycle helium re-condensing for SQUID biomagnetic measurement system. SQ-P15. ISEC 2015. July 6-9, 2015, Nagoya, Japan.

[8] Wang C. A helium re-liquefier for recovery and liquefying helium vapor from cryostat. In: Advances in Cryogenic Engineering, Vol.55A, AIP publisher, Melville, NY, 2010, pp. 687-694. [9] Wang C, Oviedo A. A helium liquefier using three 4k pulse tube crycoolers. Adv. In Cryogenic Eng., AIP Conf. Proc. 2012, 1434: 1640-1646.

[10] A.M.J. den Haan, G.H.C.J. Wijts, F. Galli, etc., Atomic resolution STM in a cryogen free dilution refrigerator at $15 \mathrm{mK}$, Rev. Sci. Instrum. 2014, 85: 035112.

[11] Uusitalo MA, Ilmoniemi RJ. Signal-space projection method for separating MEG or EEG into components. Med Biol Eng Comput. 1997, 35: 135-140 